\title{
Aplicación de la teoría de semigrupos a una ecuación de onda semilineal con disipación localizada
}

\author{
Carlos Alberto Peña Miranda ${ }^{1}$, Alfonso Pérez Salvatierrd ${ }^{2}$ y Elizabeth Cosi Cruz ${ }^{3}$
}

Resumen: En este artículo, demostramos la existencia única de la solución para la ecuación de onda semilineal con disipación localizada no lineal mediante la teoría de semigrupos.

Palabras clave: ecuación de onda semilineal; solucion débil; disipación localizada.

\section{Application of semigroup theory to a semilinear wave equation with localized dissipation}

\begin{abstract}
In this article, we demonstrate the unique existence of the solution for the semilinear wave equation with localized nonlinear dissipation using semigroup theory..
\end{abstract}

Keywords: semilinear wave equation; weak solutions; localized dissipation.

Recibido: 13/01/2019. Aceptado: 30/07/2019. Publicado online: 18/08/2020.

(C) Los autores. Este artículo es publicado por la Revista PESQUIMAT de la Facultad de Ciencias Matemáticas, Universidad Nacional Mayor de San Marcos. Este es un artículo de acceso abierto, distribuido bajo los términos de la licencia Creative Commons Atribucion-No Comercia-CompartirIgual 4.0 Internacional.(http://creativecommons.org/licenses/by-nc-sa/4.0/) que permite el uso no comercial, distribución y reproducción en cualquier medio, siempre que la obra original sea debidamente citada. Para uso comercial, por favor póngase en contacto con revistapesquimat.matematica@unmsm.edu.pe

\footnotetext{
${ }^{1}$ UNMSM, Facultad de Ciencias Matemáticas. e-mail: cpenam@unmsm.edu.pe

${ }^{2}$ UNMSM, Facultad de Ciencias Matemáticas. e-mail: aperezs@unmsm.edu.pe

${ }^{3}$ Universidad Privada Norbert Wiener, e-mail: elizabeth.cosi@uwiener.edu.pe
} 


\section{Introducción}

En el presente artículo vamos a demostrar la existencia y unicidad de solución débil para la ecuación de onda semilineal con disipación localizada no lineal dado por,

$$
u_{t t}-\Delta u+\alpha(x) u+f(u)+a(x) g\left(u_{t}\right)=0 \text { en } \Omega \times[0,+\infty[
$$

con condicion de frontera

$$
u(x, t)=0, \text { en }(x, t) \in \Gamma \times[0,+\infty[
$$

y condiciones iniciales

$$
u(x, 0)=u_{0}(x), u_{t}(x, 0)=u_{1}(x), x \in \Omega
$$

donde $\Omega$ es un conjunto abierto acotado de $\mathbb{R}^{n}, n \geq 1$ con frontera $\Gamma$ bien regular.

El objetivo del artículo es demostrar la existencia y unicidad de una solución débil para el problema (1) utilizando la teoría de semigrupos, una teoría que tuvo su avance en 1948 con la demostración del teorema de Hille - Yosida y que tiene gran importancia en el estudio de la solución a problemas de valor inicial (PVI) o problema de Cauchy abstracto, dado por

$$
\left\{\begin{array}{l}
\frac{d u}{d t}(t)=\mathcal{A} u(t) \quad t>0 \\
u(0)=u_{0} \in D(\mathcal{A}) .
\end{array}\right.
$$

Para demostrar el resultado de la existencia de soluciones, las funciones $\alpha, a, f$ y $g$, deben verificar las siguientes hipótesis

(H1) $a \in L^{\infty}(\Omega) ; a(x) \geq a_{0}>0$ casi siempre en $\Omega$.

(H2) $f \in C^{1}(\mathbb{R})$ y cumple la siguiente condición de crecimiento:

$$
|f(x)-f(y)| \leq C\left(1+|x|^{p-1}+|y|^{p-1}\right)|x-y|, \text { para todo } x, y \in \mathbb{R}
$$

para alguna constante $C>0$ y $p>1$ tal que $(n-2) p \leq n$.

(H3) La función no lineal $g: \mathbb{R} \rightarrow \mathbb{R}$ cumple

a) $g \in C^{1}(\mathbb{R})$

b) $g(s) s \geq 0, \forall s \in \mathbb{R}$

c) $g$ es una función no decreciente

d) existen constantes $m, M>0$ tal que $m|s| \leq g(s) \leq M|s|, \forall s \in \mathbb{R}$

e) $g^{\prime} \in L^{\infty}(\mathbb{R})$.

(H4) $\alpha \in W^{1, \infty}(\Omega) ; \alpha(x) \geq \alpha_{0}>0$ c.s. en $\Omega$.

El problema (1) con disipación localizada del tipo $a(x) u_{t}$ fue estudiado por Pérez, A. y Peña, C. [7, quienes demostraron la existencia y unicidad de solución regular para la ecuación de onda semilineal con disipación localizada sobre $\mathbb{R}^{n}$.

Por otro lado, la estabilización para la ecuación de la onda sujeta a disipación localizada

$$
u_{t t}-\Delta u+a(x) g\left(u_{t}\right)=0 \text { en } \Omega \times \mathbb{R}_{+}
$$


ha sido estudiado por Zuazua, E. [8] y Nakao, M. [4, 5] donde demuestra el decaimiento uniforme de las soluciones considerando la función $a$ positiva en todo el dominio $\Omega$. Además, el decaimiento uniforme de soluciones para ecuaciones de onda semilineal con disipación localizada

$$
u_{t t}-\Delta u+\alpha u+f(u)+a(x) u_{t}=0 \text { en } \Omega \times[0,+\infty[
$$

fue obtenido por Zuazua, E. [9, 10] donde asumen que $a$ es una función positiva en una vecindad $\omega$ de la frontera de $\Omega$ o en todo $\mathbb{R}$.

En la siguientes dos secciones, usando la teoría de semigrupos, demostraremos el siguiente teorema

Teorema 1.1 Si $\left\{u_{0}, u_{1}\right\} \in H_{0}^{1}(\Omega) \times L^{2}(\Omega)$ y se cumple las hipotesis (H1) - (H4), entonces existe una única solución débil del sistema (1) - (3) en la clase

$$
u \in C\left(\left[0, \infty\left[; H_{0}^{1}(\Omega)\right) \cap C^{1}\left(\left[0, \infty\left[; L^{2}(\Omega)\right) .\right.\right.\right.\right.
$$

\section{Existencia y unicidad de solución débil}

En esta sección estudiamos la existencia y unicidad de la solución débil para el sistema (1) - (3) con las hipótesis (H1) - (H4) y datos iniciales $\left\{u_{0}, u_{1}\right\} \in H_{0}^{1}(\Omega) \times L^{2}(\Omega)$.

Efectuamos el cambio de variable $v=u_{t}$ y denotamos $U=\left(\begin{array}{c}u \\ u_{t}\end{array}\right)$, el sistema 11 - 3 , se puede reescribir como el problema de Cauchy no homogéneo

$$
\left\{\begin{array}{l}
\frac{d U}{d t}+\mathcal{A} U=F(U) \\
U(0)=U_{0}
\end{array}\right.
$$

donde $F(U)=\left(\begin{array}{c}0 \\ -f(u)-a(\cdot) g\left(u_{t}\right)\end{array}\right)$ y $\mathcal{A}: D(\mathcal{A}) \subset X \rightarrow X$ es el operador definido por

$$
\mathcal{A}=\left(\begin{array}{cr}
0 & -I \\
(-\Delta+\alpha(\cdot)) I & 0
\end{array}\right)
$$

Con el fin de efectuar la formulación del semigrupo asociado al sistema (1) - (3), definimos la energía del sistema

$$
E(t):=\frac{1}{2} \int_{\Omega}\left[|\nabla u(x, t)|^{2}+\left|u_{t}(x, t)\right|^{2}+\alpha(x)|u(x, t)|^{2}\right] d x+\int_{\Omega} F(u(x, t)) d x .
$$

Observación 2.1 Por la hipótesis (H2), la función

$$
F(s)=\int_{0}^{s} f(t) d t, \forall s \in \mathbb{R}
$$

está bien definida. 
Proposición 1 La energía asociada al sistema (1) - (3), es decreciente.

Demostración. Multiplicamos la ecuación (1) por $u_{t}$ e integrando sobre $\Omega$, obtenemos

$$
\frac{1}{2} \frac{d}{d t}\left(\int_{\Omega}\left[|\nabla u|^{2}+\left|u_{t}\right|^{2}+\alpha(x)|u|^{2}\right] d x\right)+\int_{\Omega} f(u) u_{t} d x+\int_{\Omega} a(x) g\left(u_{t}\right) u_{t} d x=0 .
$$

Luego

$$
\frac{1}{2} \frac{d}{d t}\left(\int_{\Omega}\left[|\nabla u|^{2}+\left|u_{t}\right|^{2}+\alpha(x)|u|^{2}\right] d x+\int_{\Omega} F(u) d x\right)=-\int_{\Omega} a(x) g\left(u_{t}\right) u_{t} d x .
$$

De (6), hipótesis (H1) y (H3) se concluye

$$
\frac{d}{d t} E(t)=-\int_{\Omega} a(x) g\left(u_{t}\right) u_{t} d x \leq 0 .
$$

Por tanto, la energía asociada al sistema (1) - (3) es decreciente, para todo $t \in[0,+\infty[$.

Observación 2.2 Del resultado de la proposición 1, para que la energía esté bien definida, se establece que la solución $U=\left(\begin{array}{c}u \\ u_{t}\end{array}\right)$ debe satisfacer

$$
U \in H_{0}^{1}(\Omega) \times L^{2}(\Omega) .
$$

De este modo se define el espacio de fase asociado al sistema (1) - (3) dado por

$$
\mathcal{H}=H_{0}^{1}(\Omega) \times L^{2}(\Omega)
$$

con el producto interno

$$
\left\langle U_{1}, U_{2}\right\rangle_{\mathcal{H}}=\int_{\Omega}\left(\nabla u_{1} \cdot \nabla u_{2}+\alpha(x) u_{1} u_{2}+v_{1} v_{2}\right) d x
$$

donde $U_{i}=\left(\begin{array}{c}u_{i} \\ v_{i}\end{array}\right) \in \mathcal{H}$. Dotado con la norma inducida

$$
\|U\|_{\mathcal{H}}=\int_{\Omega}\left(|\nabla u|^{2}+\alpha(x)|u|^{2}+|v|^{2}\right) d x
$$

hace de $\mathcal{H}$ un espacio de Banach.

El dominio del operador $\mathcal{A}$ es el conjunto sobre el cual el operador está bien definido sobre el espacio de fase, es decir,

$$
D(\mathcal{A})=\{U \in \mathcal{H} ; \mathcal{A} U \in \mathcal{H}\}
$$

teniendo en cuenta el operador $\mathcal{A}$ definido en $\sqrt{5}$ y el vector $U=\left(\begin{array}{c}u \\ v\end{array}\right) \in \mathcal{H}$ se deduce

$$
D(\mathcal{A})=\left(H_{0}^{1}(\Omega) \cap H^{2}(\Omega)\right) \times H_{0}^{1}(\Omega) .
$$

De las inmersiones

$$
\left(H_{0}^{1}(\Omega) \cap H^{2}(\Omega)\right) \stackrel{c}{\hookrightarrow} H_{0}^{1}(\Omega) \text { y } H_{0}^{1}(\Omega) \stackrel{c}{\hookrightarrow} L^{2}(\Omega)
$$

resulta

$$
\overline{D(\mathcal{A})}=\mathcal{H}
$$


Lema 2.1 $\mathcal{A}$ es un operador disipativo.

Demostración. Sea $U=\left(\begin{array}{l}u \\ v\end{array}\right) \in D(\mathcal{A})$ y el operador $\mathcal{A}$ definido en $[5$, entonces

$$
\mathcal{A} U=\left(\begin{array}{c}
-v \\
-\Delta u+\alpha(\cdot) u
\end{array}\right) \in \mathcal{H} .
$$

Luego

$$
\begin{aligned}
\langle\mathcal{A} U, U\rangle_{\mathcal{H}} & =\int_{\Omega}[-\nabla v \cdot \nabla u-\alpha(x) v u+((-\Delta u)+\alpha(x) u) v] d x \\
& =\int_{\Omega}[-\nabla v \cdot \nabla u+(-\Delta u) v] d x
\end{aligned}
$$

aplicando la formula de Green (ver Kesavan [3] pág. 103), se obtiene

$$
\langle\mathcal{A} U, U\rangle_{\mathcal{H}}=0 \leq 0 .
$$

Por lo tanto, $\mathcal{A}$ es un operador disipativo.

Lema 2.2 $\mathcal{A}$ es un operador maximal.

Demostración. Dado $\left(\begin{array}{c}f \\ g\end{array}\right) \in \mathcal{H}$ debemos demostrar que existe una única solución $\left(\begin{array}{c}u \\ v\end{array}\right) \in D(\mathcal{A})$ tal que

$$
\left(\begin{array}{l}
u \\
v
\end{array}\right)+\mathcal{A}\left(\begin{array}{l}
u \\
v
\end{array}\right)=\left(\begin{array}{l}
f \\
g
\end{array}\right)
$$

En efecto, de la igualdad (9), se obtiene

$$
\begin{array}{r}
u-v=f \\
v-\Delta u+\alpha(\cdot) u=g
\end{array}
$$

sumando las dos ecuaciones anteriores, obtenemos

$$
-\Delta u+(\alpha(\cdot)+1) u=f+g \in L^{2}(\Omega) .
$$

A fin de garantizar la solución del sistema $(10)$, definamos la forma bilineal

$$
a: H_{0}^{1}(\Omega) \times H_{0}^{1}(\Omega) \rightarrow \mathbb{R}
$$

dada por

$$
a(u, v)=(\nabla u, \nabla v)_{L^{2}(\Omega)}+((\alpha(\cdot)+1) u, v)_{L^{2}(\Omega)} .
$$


Afirmación 2.1 La forma bilineal $a(u, v)$ definido en (11) es continua y coerciva.

En efecto,

i) $a(u, v)$ es continua.

En efecto, sea $u, v \in H_{0}^{1}(\Omega)$, aplicando la desigualdad de Cauchy - Schwarz se obtiene

$$
\begin{aligned}
|a(u, v)| & \leq|\nabla u|_{L^{2}(\Omega)}|\nabla v|_{L^{2}(\Omega)}+\left(1+|\alpha|_{\infty}\right)|u|_{L^{2}(\Omega)}|v|_{L^{2}(\Omega)} \\
& =|u|_{H_{0}^{1}(\Omega)}|v|_{H_{0}^{1}(\Omega)}+\left(1+|\alpha|_{\infty}\right)|u|_{L^{2}(\Omega)}|v|_{L^{2}(\Omega)} .
\end{aligned}
$$

De la inmersión de Sobolev $H_{0}^{1}(\Omega) \stackrel{c}{\hookrightarrow} L^{2}(\Omega)$, existe una constante $\widehat{C}>0$ tal que

$$
|u|_{L^{2}(\Omega)} \leq \widehat{C}|u|_{H_{0}^{1}(\Omega)} \text {, para todo } u \in H_{0}^{1}(\Omega) .
$$

De (12) y (13) obtenemos

$$
\begin{aligned}
|a(u, v)| & \leq|u|_{H_{0}^{1}(\Omega)}|v|_{H_{0}^{1}(\Omega)}+\widehat{C}^{2}\left(1+|\alpha|_{\infty}\right)|u|_{H_{0}^{1}(\Omega)}|v|_{H_{0}^{1}(\Omega)} \\
& \leq 2 \tilde{C}|u|_{H_{0}^{1}(\Omega)}|v|_{H_{0}^{1}(\Omega)} .
\end{aligned}
$$

donde $\tilde{C}=\operatorname{máx}\left\{1, \widehat{C}^{2}\left(1+|\alpha|_{\infty}\right)\right\}$.

ii) $a(u, v)$ es coerciva.

En efecto, sea $u \in H_{0}^{1}(\Omega)$, de la hipótesis (H4) y (11), resulta

$$
\begin{aligned}
|a(u, u)| & =|\nabla u|_{L^{2}(\Omega)}^{2}+|u|_{L^{2}(\Omega)}^{2}+|(\alpha(\cdot) u, u)| \\
& \geq|\nabla u|_{L^{2}(\Omega)}^{2}+|u|_{L^{2}(\Omega)}^{2}+\alpha_{0}|u|_{L^{2}(\Omega)}^{2} \\
& \geq k\left(|\nabla u|_{L^{2}(\Omega)}^{2}+|u|_{L^{2}(\Omega)}^{2}\right) \\
& \geq k|u|_{H_{0}^{1}(\Omega)}^{2}
\end{aligned}
$$

donde $k=\operatorname{mín}\left\{1,1+\alpha_{0}\right\}$.

Con lo que queda demostrado la afirmación 2.1.

Dado $\psi \in L^{2}(\Omega)$, definimos la siguiente aplicación

$$
\begin{aligned}
L: H_{0}^{1}(\Omega) & \rightarrow \mathbb{R} \\
v & \mapsto\langle L, v\rangle:=(\psi, v)
\end{aligned}
$$

entonces $L$ es una forma lineal y continua, es decir, $L \in\left(H_{0}^{1}(\Omega)\right)^{\prime}$. Por teorema de Lax - Milgram (ver Brezis [2] pág. 140), la ecuación 10 posee una única solución $u \in H_{0}^{1}(\Omega)$ tal que

$$
a(u, v)=\langle L, v\rangle:=(\psi, v)_{L^{2}(\Omega)}, \forall v \in H_{0}^{1}(\Omega) .
$$

Para todo $\varphi \in D(\Omega)$, tenemos

$$
\begin{aligned}
(\psi, \varphi)_{L^{2}(\Omega)} & =\langle L, \varphi\rangle \\
& =a(u, \varphi) \\
& =(\nabla u, \nabla \varphi)+((\alpha(\cdot)+1) u, \varphi) \\
& =(-\Delta u, \varphi)+((\alpha(\cdot)+1) u, \varphi) \\
& =(-\Delta u+(\alpha(\cdot)+1) u, \varphi)
\end{aligned}
$$


luego $-\Delta u+(\alpha(\cdot)+1) u=\psi \in L^{2}(\Omega)$ y por el teorema 3.3.3 (ver Kesavan [3] pág. 139) $u \in H^{2}(\Omega)$.

Por lo tanto, dado $\left(\begin{array}{c}f \\ g\end{array}\right) \in \mathcal{H}=H_{0}^{1}(\Omega) \times L^{2}(\Omega)$ existe una única $\left(\begin{array}{c}u \\ v\end{array}\right) \in D(\mathcal{A})$ tal que

$$
\left(\begin{array}{l}
u \\
v
\end{array}\right)+\mathcal{A}\left(\begin{array}{l}
u \\
v
\end{array}\right)=\left(\begin{array}{l}
f \\
g
\end{array}\right)
$$

y esto demuestra que $\mathcal{A}$ es maximal.

Lema 2.3 El operador $F: \mathcal{H} \rightarrow \mathcal{H}$ dado por

$$
F(U)=\left(\begin{array}{c}
0 \\
-f(u)-a(\cdot) g(v)
\end{array}\right) \text { para todo } U=\left(\begin{array}{l}
u \\
v
\end{array}\right) \in \mathcal{H}
$$

está bien definida.

Demostración. Sea $U=\left(\begin{array}{l}u \\ v\end{array}\right) \in \mathcal{H}$ sólo nos faltaría demostrar que

$$
f(u)+a(\cdot) g(v) \in L^{2}(\Omega) .
$$

En efecto, por las hipótesis (H2) y de la desigualdad $(a+b)^{2} \leq 2\left(a^{2}+b^{2}\right)$ se tiene

$$
\begin{aligned}
\int_{\Omega}|f(u)|^{2} d x & \leq \int_{\Omega}\left[\left(1+|u|^{p-1}\right)|u|\right]^{2} d x \\
& \leq 2 C\left(\int_{\Omega}|u|^{2} d x+\int_{\Omega}|u|^{2 p} d x\right) \\
& =2 C\left(|u|_{L^{2}(\Omega)}^{2}+|u|_{L^{2 p}(\Omega)}^{2 p}\right) .
\end{aligned}
$$

De la inmersión de Sobolev $H_{0}^{1}(\Omega) \hookrightarrow L^{2 p}(\Omega)$, existe una constante $\tilde{C}$ tal que

$$
|u|_{L^{2 p}(\Omega)} \leq \tilde{C}|u|_{H_{0}^{1}(\Omega)}, \text { para todo } u \in H_{0}^{1}(\Omega) .
$$

De las desigualdades (14) y 15 resulta

$$
\int_{\Omega}|f(u)|^{2} d x \leq 2 C\left(|u|_{L^{2}(\Omega)}^{2}+\tilde{C}^{2}|u|_{H_{0}^{1}(\Omega)}^{2 p}\right)<\infty .
$$

Además de la hipótesis (H1) y (H3) obtenemos

$$
\int_{\Omega}|a(x) g(v)|^{2} d x \leq M^{2}|a|_{\infty}^{2} \int_{\Omega}|v|^{2} d x<\infty .
$$

De (16) y (17) se prueba la buena definición de $F$.

Lema 2.4 El operador $F: \mathcal{H} \rightarrow \mathcal{H}$ es localmente lipschitziano.

Demostración. Sean $U_{1}=\left(\begin{array}{c}u_{1} \\ v_{1}\end{array}\right) \in \mathcal{H}$ y $U_{2}=\left(\begin{array}{c}u_{2} \\ v_{2}\end{array}\right) \in \mathcal{H}$ tal que

$$
\left\|U_{1}\right\|_{\mathcal{H}} \leq M \text { y }\left\|U_{2}\right\|_{\mathcal{H}} \leq M \text { para algún } M>0 .
$$

Por demostrar que existe una constante $L_{M}>0$ tal que

$$
\left\|F\left(U_{1}\right)-F\left(U_{2}\right)\right\|_{\mathcal{H}} \leq L_{M}\left\|U_{1}-U_{2}\right\|_{\mathcal{H}} .
$$


En efecto, por las hipótesis (H2) y de la desigualdad $(a+b+c)^{2} \leq 3\left(a^{2}+b^{2}+c^{2}\right)$ resulta

$$
\begin{aligned}
\left|f\left(u_{1}\right)-f\left(u_{2}\right)\right|_{L^{2}(\Omega)}^{2} & \leq C^{2} \int_{\Omega}\left\{\left(1+\left|u_{1}\right|^{p-1}+\left|u_{2}\right|^{p-1}\right)\left|u_{1}-u_{2}\right|\right\}^{2} d x \\
& \leq 3 C^{2}\left(\int_{\Omega}\left|u_{1}-u_{2}\right|^{2} d x+\int_{\Omega}\left|u_{1}\right|^{2(p-1)}\left|u_{1}-u_{2}\right|^{2} d x\right. \\
& \left.\quad+\int_{\Omega}\left|u_{2}\right|^{2(p-1)}\left|u_{1}-u_{2}\right|^{2} d x\right) .
\end{aligned}
$$

Desde que $\frac{1}{p}+\frac{p-1}{p}=1$, usando la desigualdad de Hölder (ver Adams [1] pág. 24) obtenemos

$$
\begin{aligned}
\left|f\left(u_{1}\right)-f\left(u_{2}\right)\right|_{L^{2}(\Omega)}^{2} \leq 3 C^{2}\left(\left|u_{1}-u_{2}\right|_{L^{2}(\Omega)}^{2}+\left|u_{1}\right|_{L^{2 p}(\Omega)}^{2(p-1)}\left|u_{1}-u_{2}\right|_{L^{2 p}(\Omega)}^{2}\right. & \\
& \left.+\left|u_{2}\right|_{L^{2 p}(\Omega)}^{2(p-1)}\left|u_{1}-u_{2}\right|_{L^{2 p}(\Omega)}^{2}\right) .
\end{aligned}
$$

De las desigualdades (13), (15) y (18) obtenemos

$$
\begin{aligned}
\left|f\left(u_{1}\right)-f\left(u_{2}\right)\right|_{L^{2}(\Omega)}^{2} \leq 3 C^{2}\left(\widehat{C}^{2}\left|u_{1}-u_{2}\right|_{H_{0}^{1}(\Omega)}^{2}+\tilde{C}^{2 p} M^{2(p-1)}\left|u_{1}-u_{2}\right|_{H_{0}^{1}(\Omega)}^{2}\right. & \\
& \left.+\tilde{C}^{2 p} M^{2(p-1)}\left|u_{1}-u_{2}\right|_{H_{0}^{1}(\Omega)}^{2}\right) .
\end{aligned}
$$

Luego

$$
\left|f\left(u_{1}\right)-f\left(u_{2}\right)\right|_{L^{2}(\Omega)}^{2} \leq C_{M}\left|u_{1}-u_{2}\right|_{H_{0}^{1}(\Omega)}^{2}
$$

donde $C_{M}=3 C^{2}\left(\widehat{C}^{2}+\tilde{C}^{2 p} M^{2(p-1)}+\tilde{C}^{2 p} M^{2(p-1)}\right)$.

De la hipótesis (H3), por el teorema del valor medio, existe $\lambda \in\left(s_{1}, s_{2}\right)$ tal que

$$
\left|g\left(s_{1}\right)-g\left(s_{2}\right)\right|=\left|g^{\prime}(\lambda)\right|\left|s_{1}-s_{2}\right| \leq\left|g^{\prime}\right|_{L^{\infty}(\Omega)}\left|s_{1}-s_{2}\right| .
$$

Usando 20 se obtiene

$$
\left|a(\cdot)\left(g\left(v_{1}\right)-g\left(v_{2}\right)\right)\right|_{L^{2}(\Omega)}^{2} \leq\left|g^{\prime}\right|_{L^{\infty}(\Omega)}^{2}|a|_{L^{\infty}(\Omega)}^{2}\left|v_{1}-v_{2}\right|_{L^{2}(\Omega)}^{2}
$$

De las desigualdades (19) y (21) resulta

$$
\begin{aligned}
\left\|F\left(U_{1}\right)-F\left(U_{2}\right)\right\|_{\mathcal{H}} & =\left|f\left(u_{1}\right)-f\left(u_{2}\right)\right|_{L^{2}(\Omega)}^{2}+\left|a(\cdot)\left(g\left(v_{1}\right)-g\left(v_{2}\right)\right)\right|_{L^{2}(\Omega)}^{2} \\
& \leq C_{M}\left|u_{1}-u_{2}\right|_{H_{0}^{1}(\Omega)}^{2}+\left|g^{\prime}\right|_{L^{\infty}(\Omega)}^{2}|a|_{L^{\infty}(\Omega)}^{2}\left|v_{1}-v_{2}\right|_{L^{2}(\Omega)}^{2} \\
& \leq L_{M}\left(\left|u_{1}-u_{2}\right|_{H_{0}^{1}(\Omega)}^{2}+\left|v_{1}-v_{2}\right|_{L^{2}(\Omega)}^{2}\right) \\
& \leq L_{M}\left\|U_{1}-U_{2}\right\|_{\mathcal{H}},
\end{aligned}
$$

donde $L_{M}=\operatorname{máx}\left\{C_{M},\left|g^{\prime}\right|_{L^{\infty}(\Omega)}^{2}|a|_{L^{\infty}(\Omega)}^{2}\right\}$.

Finalmente, como $F$ es continua y localmente lipschitziana, por el teorema 1.4 (ver Pazy [6] pág. 185) para cada $U_{0}=\left(\begin{array}{l}u_{0} \\ v_{0}\end{array}\right) \in \mathcal{H}$, existe una única solución de sistema 44 en la clase

$$
U=\left(\begin{array}{c}
u \\
u_{t}
\end{array}\right) \in C\left(\left[0, t_{\max }[; \mathcal{H})\right.\right.
$$

es decir,

$$
u \in C\left(\left[0, t_{\max }\left[; H_{0}^{1}(\Omega)\right) \cap C^{1}\left(\left[0, t_{\max }\left[; L^{2}(\Omega)\right) .\right.\right.\right.\right.
$$




\section{Prolongamiento de la solución débil}

En esta sección obtendremos la solución global del problema (1); para ello extenderemos nuestra solución obtenida anteriormente, aplicando el teorema 1.4 (ver Pazy [6] pág. 185).

Integrando (8) desde $t_{1}$ hasta $t_{2}$ se obtiene

$$
E\left(t_{2}\right)-E\left(t_{1}\right)=-\int_{t_{1}}^{t_{2}} \int_{\mathbb{R}^{n}} a(x)\left|u_{t}(x, t)\right|^{2} d x d t, \text { para todo } t_{2}>t_{1} \geq 0 .
$$

Por lo tanto

$$
E\left(t_{2}\right) \leq E\left(t_{1}\right), \text { para todo } t_{2}>t_{1} \geq 0,
$$

lo que demuestra que la energía asociada al sistema (1) - (3) es decreciente, para todo $t \in[0,+\infty[$.

Afirmación $3.1 t_{\max }=+\infty$.

En efecto, si suponemos que $t_{\max }<+\infty$ entonces por el teorema 1.4 (ver Pazy [6] pág. 185) se tiene

$$
\lim _{t \rightarrow t_{\max }}\|U(t)\|_{\mathcal{H}}=+\infty
$$

Pero

$$
\begin{aligned}
\|U(t)\|_{\mathcal{H}}=\int_{\Omega}\left[|\nabla u|^{2}+\left|u_{t}\right|^{2}+\alpha(x)|u|^{2}\right] d x & \leq E(t) \\
& \leq E(0)<\infty
\end{aligned}
$$

lo cual es absurdo. Por lo tanto $t_{\max }=+\infty$, es decir, la solución débil existen en todo el intervalo $[0,+\infty[$.

\section{Conclusión}

La teoría de semigrupos demostró ser una herramienta poderosa en la demostración de existencia y unicidad de solución del problema (1) - (3). Además, tal teoría se puede aplicar a cualquier tipo de ecuaciones diferenciales parciales de evolución ya sea que tengan términos lineales y no lineales, como en nuestro caso que se tiene un término localmente disipativo no lineal

\section{Referencias bibliográficas}

[1] Adams, R. A. (1975). Sobolev Spaces. New York, States United: Academic Press.

[2] Brezis, H. (2010). Functional Analysis, Sobolev Spaces and Partial Differential Equations. New York, States United: Springer.

[3] Kesavan, S.(1989). Topics in functional analysis and applications. Bangalore, India: Wiley Eastern Limited.

[4] Nakao, M. (1978). A difference inequality and its applications to nonlinear evolution equations. Journal of the Mathematical Society of Japan 30(4), 747-762.

[5] Nakao, M. (1996). Decay of solutions of the wave equation with a local degenerate dissipation. Israel Journal of Mathematics, 95, 25-42. 
[6] Pazy, A. (1983). Semigroups of Linear Operators and Applications to Partial Differential Equations, New York, States United: Springer-Verlag.

[7] Pérez, A. y Peña, C. (2012). Existencia y unicidad de soluciones regulares de la ecuación de onda semilineal con disipación localizada en $\mathbb{R}^{n}$. Pesquimat, 15(2), 51-60.

[8] Zuazua, E. (1990). Exponential decay for the semilinear wave equations, with locally distributed damping. Communications in Partial Differential Equations, 15(2), 205-235.

[9] Zuazua, E. (1991). Exponential decay for the semilinear wave equations, with localized Damping in Unbounded Domains. Journal de Mathématiques Pures et Appliquées, 70 , $513-529$.

[10] Zuazua, E. (1988). Stability and decay for a class of nonlinear hyperbolic problems, Asymptotic Analysis, 1(2), 161-185. 